\title{
Magnetic detonation structure in crystals of nanomagnets controlled by thermal conduction and volume viscosity
}

\author{
O. Jukimenko, ${ }^{1}$ M. Modestov, ${ }^{2}$ M. Marklund, ${ }^{1,3}$ and V. Bychkov ${ }^{1}$ \\ ${ }^{1}$ Department of Physics, Umeå University, SE-901 87 Umeå, Sweden \\ ${ }^{2}$ Nordita, AlbaNova University Center, SE-106 91 Stockholm, Sweden \\ ${ }^{3}$ Department of Applied Physics, Chalmers University of Technology, SE-412 96 Göteborg, Sweden \\ (Received 17 December 2014; revised manuscript received 17 March 2015; published 25 March 2015)
}

\begin{abstract}
Experimentally detected ultrafast spin avalanches spreading in crystals of molecular (nano)magnets [Decelle et al., Phys. Rev. Lett. 102, 027203 (2009)] have recently been explained in terms of magnetic detonation [Modestov et al., Phys. Rev. Lett. 107, 207208 (2011)]. Here magnetic detonation structure is investigated by taking into account transport processes of the crystals such as thermal conduction and volume viscosity. The transport processes result in smooth profiles of the most important thermodynamical crystal parameters, temperature, density, and pressure, all over the magnetic detonation front, including the leading shock, which is one of the key regions of magnetic detonation. In the case of zero volume viscosity, thermal conduction leads to an isothermal discontinuity instead of the shock, for which temperature is continuous while density and pressure experience jump. It is also demonstrated that the thickness of the magnetic detonation front may be controlled by applying the transverse-magnetic field, which is important for possible experimental observations of magnetic detonation.

DOI: 10.1103/PhysRevB.91.094428

PACS number(s): 75.50.Xx, 47.40.Rs, 47.70.Pq, 75.60.Jk
\end{abstract}

\section{INTRODUCTION}

Presently, there is much interest in molecular (nano)magnets with unique superparamagnetic properties, which may be used for quantum computing and memory storage [1-6]. A remarkable feature of nanomagnets is that, in contrast to classical magnets, these macromolecules with large effective molecular spin (e.g., $S=10$ for $\mathrm{Mn}_{12}$ acetate) can keep their spin orientation upon the reversal of the external magnetic field $[7,8]$. Because of the strong molecular anisotropy, the spin of a nanomagnet is directed preferentially along the so-called easy axis, and it leads to a considerable energy barrier between the spin-up and spin-down states. At low temperatures, in a magnetic field directed along the easy axis, the states with spin along the field and against the field become stable and metastable, respectively. The energy difference between the two states is determined by the Zeeman energy $Q$, as illustrated in Fig. 1, with the energy barrier designated by $E_{a}$. The barrier hinders spontaneous transition from the metastable to stable state at low temperatures [9-13], so that fast spin flipping requires help from outside.

For nanomagnets composing a crystal, relatively fast spin flipping of one particular molecule may be induced by energy supplied by its neighbors. When all or most of the molecules of a crystal are initially in the metastable state, then local heating by an external source may trigger local spin flipping, with Zeeman energy released in the heated region and transported to the next layer of the crystal [3-6,14-18]. The heat facilitates spin flipping in the next layer and so on, so that the process spreads in a crystal as a thin, self-supporting magnetization front, spin avalanche, well localized spatially. Usually, energy in spin avalanches is transported from one crystal layer to another by means of thermal conduction, and hence a spin avalanche propagates at moderate speed, $\sim 1-10 \mathrm{~m} / \mathrm{s}$ [3-6]. Due to the striking similarity of such avalanches to slow combustion flame, deflagration, avalanches of this type are typically called magnetic deflagration.
In contrast to the slow magnetic deflagration studied in the absolute majority of works on the subject [3-6,14-18], recent experiments presented in Ref. [19] detected ultrafast spin avalanches propagating at a speed comparable to the sound speed in the crystals, $\approx 2000 \mathrm{~m} / \mathrm{s}$. The theory presented in Ref. [20] has explained the ultrafast spin avalanches in terms of "magnetic detonation" and has investigated the key properties of the process. In particular, it has been demonstrated that magnetic detonation belongs to the type of weak detonations and propagates with speed only slightly exceeding the sound speed, $\approx 2000 \mathrm{~m} / \mathrm{s}$. Reference [20] has also studied the structure of magnetic detonation within the traditional combustion model of a detonation front consisting of an infinitely thin leading shock and a zone of energy release of finite thickness [21]. Such a model was originally developed for gaseous detonations, which are quite strong and propagate with speed exceeding the sound speed in gases by an order of magnitude. However, applying such a model to magnetic detonation, which is extremely weak by combustion scales (although quite strong when compared to magnetic deflagration), is not rigorous. In contrast to strong shocks in gases, which are infinitely thin from the hydrodynamic point of view, a weak shock exhibits a continuous structure controlled by transport processes such as thermal conduction and/or volume viscosity [22]. The same property should be naturally expected for magnetic detonation.

The purpose of the present work is to provide an accurate description of the magnetic detonation structure in crystals of nanomagnets by taking into account thermal conduction and volume viscosity. We point out that, unlike commonly known shear viscosity arising due to the relative motion of gas or fluid layers, volume viscosity describes momentum and energy dissipations due to compression of a medium. While shear viscosity is not typical for solid-state processes such as magnetic detonation in the crystals of nanomagnets, volume viscosity has to be considered. Here we show that, in contrast to the previously suggested model of Ref. [20], the transport 
(a)
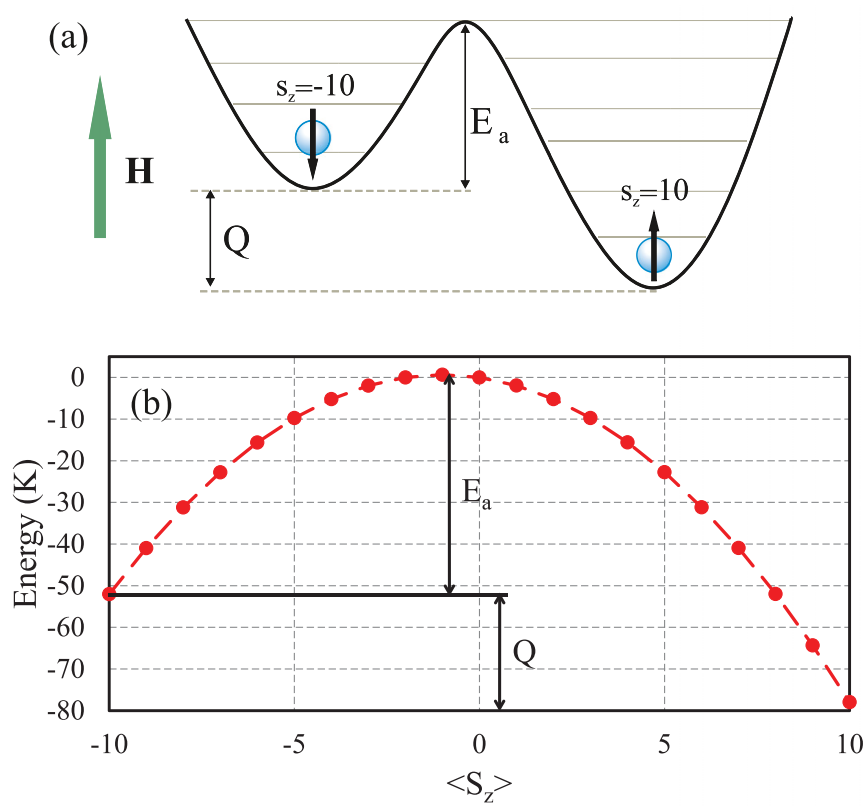

FIG. 1. (Color online) (a) Schematic representation of the double-well structure of a nanomagnet. (b) Energy levels for $\mathrm{Mn}_{12}$ acetate in the external magnetic field $B_{z}=1 \mathrm{~T}$. Axis $z$ is parallel to the easy axis of the crystal. The energy barrier (activation energy) $E_{a}$ and the Zeeman energy $Q$ are indicated.

processes result in smooth profiles of the most important thermodynamical crystal parameters, such as temperature, density, and pressure, all over the magnetic detonation front, including the leading weak shock, which is one of the key regions of magnetic detonation. In the case of zero volume viscosity, however, thermal conduction leads to an isothermal discontinuity instead of the shock, for which temperature is continuous while density and pressure experience jump.

The present paper is organized as follows. In Sec. II we give an overview of basic features of gaseous combustion detonation needed for proper understanding of magnetic detonation in crystals of nanomagnets. In Sec. III we present the basic equations describing magnetic detonation, develop the analytical theory for the most important detonation parameters, and study the magnetic detonation structure controlled only by thermal conduction assuming zero volume viscosity; such a structure involves isothermal discontinuity instead of the shock employed in Ref. [20]. In Sec. IV we demonstrate dramatic modifications of the magnetic detonation structure due to volume viscosity.

\section{BASIC FEATURES OF GASEOUS COMBUSTION DETONATION}

In this section we discuss briefly the most important features and the methods of investigation of traditional gaseous combustion detonations in order to highlight the similarity and difference between combustion and magnetic detonations. By definition, detonation is a fast supersonic combustion regime, for which preheating of the cold fuel mixture happens due to the leading shock (see Fig. 2). In turn, shock propagation without decay in combustion detonation is supported by energy release in chemical reactions in the active reaction
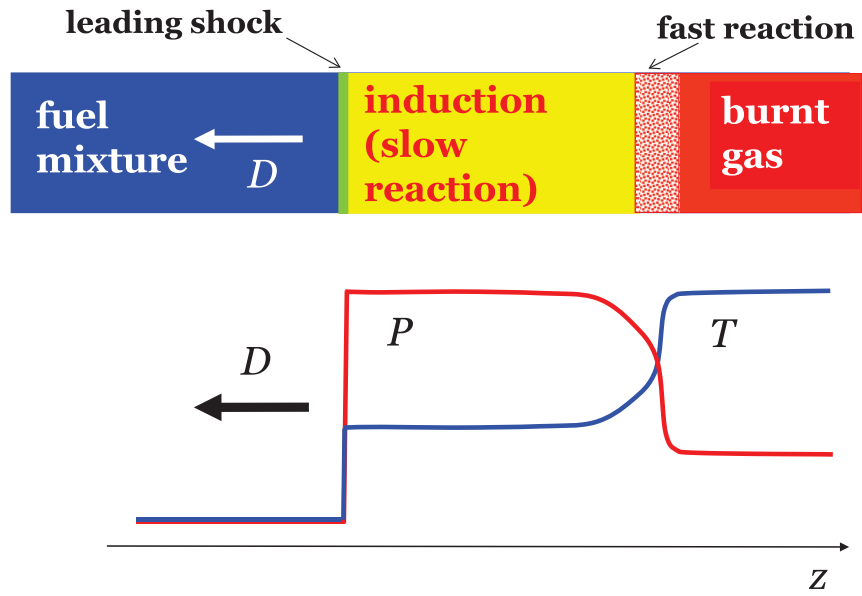

FIG. 2. (Color online) Schematic of strong gaseous detonation with characteristic profiles of temperature and pressure.

zone. Typically, for combustion, the activation energy of the reactions is quite high, so that reactions develop relatively slowly just after the leading shock in the so-called induction zone (although much faster than in the fuel mixture). A certain (induction) time is required for self-acceleration of chemical reactions in a gas parcel after passing the shock, after which the chemical reactions become fast and convert the fuel mixture into the burning products with the release of a large amount of energy and strong expansion of the burning gas.

Here we are interested in a planar stationary onedimensional detonation propagating with constant supersonic speed $D$ in a uniform gaseous mixture; we take the detonation front propagating along the $z$ axis in the negative direction. By adopting the reference frame of the stationary detonation front, we obtain the fuel mixture moving with velocity $u_{0}=D$ towards the leading shock of zero thickness; the initial density, pressure, and temperature are designated by $\rho_{0}, P_{0}$, and $T_{0}$. The fuel mixture is compressed in the shock (labeled $s$ ) with a strong increase in density, pressure, and temperature to $\rho_{s}>\rho_{0}, P_{s}>P_{0}, T_{s}>T_{0}$ and with a drop in the gas velocity to a new subsonic value, $u_{s}<D$. The temperature increase initiates combustion reactions with the release of chemical energy, leading to an even stronger temperature increase until it reaches the final maximal value in the detonation products (labeled $d$ ), $T_{d}>T_{s}$. At the same time, pressure and density decrease in the reaction region, $\rho_{d}<\rho_{s}, P_{d}<P_{s}$, as illustrated schematically in Fig. 2.

Modifications of the gas parameters in a detonation front are described by the hydrodynamic laws of mass, momentum, and energy conservation expressed in terms of fluxes [22]:

$$
\begin{gathered}
\rho u=\text { const }_{1}=\rho_{0} D, \\
P+\rho u^{2}=\text { const }_{2}=P_{0}+\rho_{0} D^{2}, \\
\rho u\left(h+\frac{u^{2}}{2}\right)=\text { const }_{3}=\rho_{0} D\left(h_{0}+\frac{D^{2}}{2}\right),
\end{gathered}
$$

where $h$ is the gas enthalpy,

$$
h=\frac{\gamma P}{(\gamma-1) \rho}+a Q,
$$




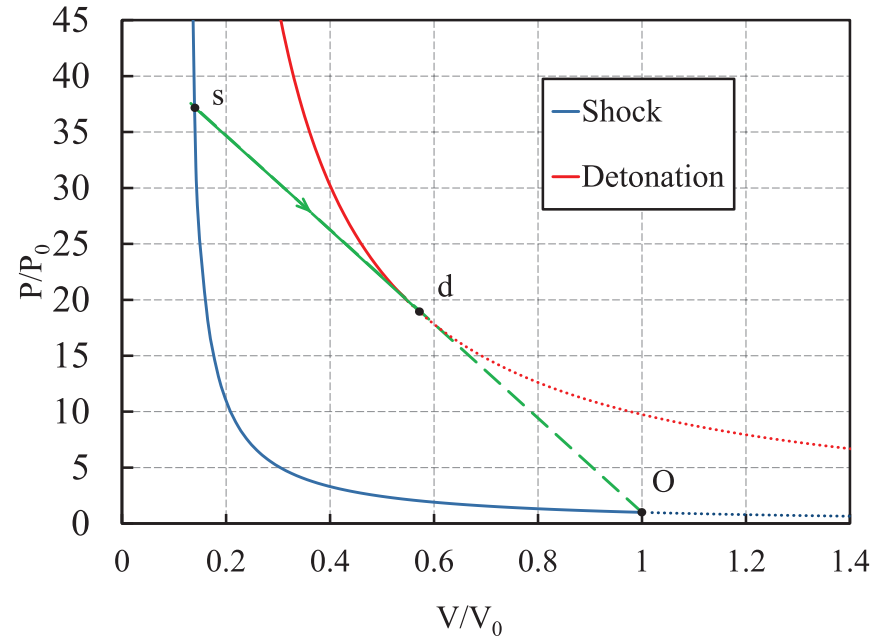

FIG. 3. (Color online) The pressure-volume diagram for shock (blue curve) and detonation (red curve) in a gas mixture. The scaled energy release is $(\gamma-1) Q / \gamma P_{0} V_{0}=9$, with the initial volume per unit mass $V_{0}=6.33 \mathrm{~m}^{3} / \mathrm{kg}$ and initial pressure $P_{0}=1.33 \times 10^{4} \mathrm{~Pa}$. The green straight line shows the transition from the shock point $s$ to the combustion products $d$.

$\gamma$ is the adiabatic exponent, $Q$ is the chemical energy stored per unit mass, and $a$ is the fraction of the unburned fuel mixture, which changes from $a_{0}=a_{s}=1$ in the fresh and shocked gas to $a_{d}=0$ in the detonation products. The hydrodynamic equations (1)-(3) are complemented by the equation of state, which is taken for gaseous detonations in the form of the ideal-gas law,

$$
P=\frac{\rho}{m} R T,
$$

where $R$ is the ideal-gas constant and $m$ is the molar mass of the gas (for simplicity we assume here that the molar mass does not change in the combustion process). Traditionally, the theory of shock waves and detonations employs volume per unit mass $V \equiv 1 / \rho$ to analyze the process [22].

Modifications of the gas parameters in a leading shock are described by Eqs. (1)-(3) with zero energy release in the reaction within the discontinuous shock, $a_{s}=1$. Then Eqs. (1)-(3) may be reduced to the so-called Hugoniot equation, which specifies all possible finite states of the shocked gas $P_{s}, V_{s}$ for a fixed initial state $P_{0}, V_{0}$; the result is demonstrated by the blue solid curve in Fig. 3 for the initial state corresponding to the ideal gas at initial volume per unit mass $V_{0}=6.33 \mathrm{~m}^{3} / \mathrm{kg}$ and pressure $P_{0}=1.33 \times 10^{4} \mathrm{~Pa}$. For any particular final state $s$ at the shock, Eqs. (1) and (2) relate the slope of the green straight line $(s O)$ in Fig. 3 to the front propagation speed $D$ as [22]

$$
\rho_{0}^{2} D^{2}=\frac{P_{s}-P_{0}}{V_{0}-V_{s}} .
$$

In order to obtain the final state of the detonation products we solve Eqs. (1)-(3) for the case of complete burning $a_{d}=0$; the result is plotted by the solid red curve in Fig. 3 for the scaled energy release $(\gamma-1) Q /\left(\gamma P_{0} V_{0}\right)=9$, corresponding to acetylene-air combustion [23]. The red curve (for detonation) corresponds to higher pressure for the same volume than

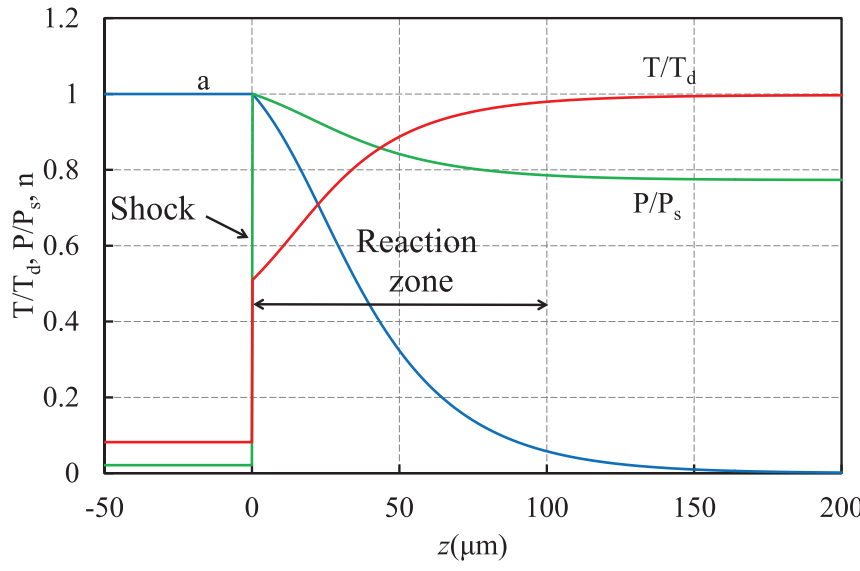

FIG. 4. (Color online) Characteristic detonation structure in a gaseous mixture with parameters representing acetylene-air combustion. The scaling is performed by using pressure at the shock $P_{s}=$ 3.7 MPa and the temperature in the detonation products $T_{d}=2988 \mathrm{~K}$; other computational parameters are specified in the text.

the blue curve (for a shock) due to the energy release in a detonation front. Still, unlike a shock, detonation speed is not a free parameter, but it is determined by particular boundary conditions. For example, the case of a freely propagating detonation corresponds to the so-called Chapman-Jouguet (CJ) detonation regime, with the detonation products moving away from the front at local sound speed [22]. The CJ detonation propagates with the lowest speed possible for detonations for a fixed energy release and is indicated by the green tangent line in Fig. 3. Thus, on the pressure-volume diagram, the CJ detonation structure corresponds first to the jump from point $O$ (the initial state) to point $s$ at the shock and then the reaction development behind the shock along the green straight line from point $s$ to point $d$ (detonation products). It may be shown that the CJ detonation speed is determined by the energy release in the combustion process [22].

The internal structure of the reaction zone in the detonation front (transition from point $s$ to point $d$ in Fig. 3) is specified by reaction kinetics of a particular fuel mixture. Here we illustrate the detonation structure for the simplified model of acetylene-air combustion described by a single one-step Arrhenius reaction [23],

$$
\frac{\partial a}{\partial t}=-K \rho a \exp \left(-E_{a} / T\right)
$$

where $K=10^{9} \mathrm{~m}^{3} \mathrm{~kg}^{-1} \mathrm{~s}^{-1}$ is a preexponential factor and $E_{a} / T_{0}=29.3$ is the scaled activation energy. Then the stationary detonation structure is determined by Eq. (7) rewritten in the reference frame of the front as

$$
u \frac{\partial a}{\partial z}=K \rho a \exp \left(-E_{a} / T\right) .
$$

By solving Eq. (8) together with Eqs. (1)-(3) we find the detonation front structure as shown in Fig. 4. To obtain the plots we have used the scaled energy release $(\gamma-1) Q /\left(\gamma P_{0} V_{0}\right)=9$, with the initial gas pressure $P_{0}=1.33 \times 10^{4}$ and temperature $T_{0}=293 \mathrm{~K}$. Then by using conservation laws (1)-(3) one finds the maximal pressure in the detonation front achieved at the shock is $P_{s}=3.7 \mathrm{MPa}$ and the maximal temperature achieved 
in the detonation products $T_{d}=2988 \mathrm{~K}$; these two values have been used for scaling in Fig. 4. As we can see, Fig. 4 presents a detonation structure similar to the qualitative sketch in Fig. 2, although the induction length is not exhibited so strongly by the acetylene-air detonations.

\section{MAGNETIC DETONATION STRUCTURE DUE TO HEAT TRANSFER}

\section{A. Basic equations}

The theory of combustion detonation summarized briefly in the previous section provides the clue for describing the magnetic detonation in crystals of nanomagnets; conceptually, the same model has been employed for the analysis of magnetic detonation in Ref. [20]. Still, here we stress important differences in some combustion and magnetic detonation properties, which are taken into account below. First, magnetic detonation in crystals of nanomagnets is extremely weak, so it propagates with speed only slightly exceeding the sound speed $c_{0}$ in the crystal, $D \approx c_{0}$. For that reason, in stark contrast to combustion detonations, the leading shock in magnetic detonation is not a discontinuity but a smooth transition region with structure determined by transport processes, namely, thermal conduction $\kappa$ and volume viscosity $\eta$, which requires proper modifications of conservation laws (1)-(3) at the detonation front.

So far the theoretical models of spin avalanches in crystals of nanomagnets have taken into account only thermal conduction and have neglected volume viscosity [14-17]; in this section we use the same approach and consider the influence of only thermal conduction. Volume viscosity will be taken into account in the next section. Although we deal with the solid state, propagation of shocks and detonations in crystals of nanomagnets are also described by hydrodynamic conservation laws of mass, momentum, and energy similar to Eqs. (1)-(3) (see Refs. [20,24]):

$$
\begin{gathered}
\rho u=\rho_{0} D, \\
P+\rho u^{2}=P_{0}+\rho_{0} D^{2}, \\
\rho u\left(\varepsilon+Q a+\frac{P}{\rho}+\frac{u^{2}}{2}\right)-\kappa \frac{d T}{d z} \\
=\rho_{0} D\left(\varepsilon_{0}+Q+\frac{P_{0}}{\rho_{0}}+\frac{D^{2}}{2}\right),
\end{gathered}
$$

where $\varepsilon$ is the thermal energy per mass unit, $Q$ stands for Zeeman energy per unit mass, and $a$ is the fraction of molecules in the metastable state. Zeeman energy $Q$ and the activation energy $E_{a}$ are determined by the applied magnetic field and can be obtained by using a Hamiltonian for the $\mathrm{Mn}_{12}$ acetate molecule [25],

$$
\mathcal{H}=-D_{a} S_{z}^{2}-g \mu_{B} B_{z} S_{z}
$$

where $B_{z}$ is the magnetic field, $g=1.94$ is the gyromagnetic factor, $\mu_{B}$ is the Bohr magneton, and $D_{a}=0.65 \mathrm{~K}$ is the magnetic anisotropy constant. We assume that the field is applied along the easy axis ( $z$ axis). The Zeeman energy is found as the energy difference between the stable, $S_{z}=10$, and metastable, $S_{z}=-10$, states of the nanomagnet, as illustrated

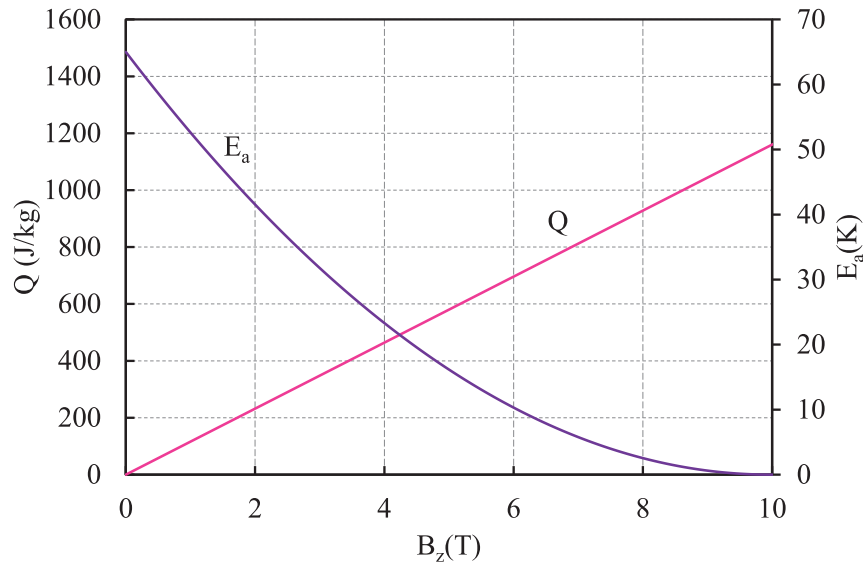

FIG. 5. (Color online) The Zeeman energy $Q$ and activation $E_{a}$ vs the external magnetic field $B_{z}$.

in Fig. 1:

$$
Q=2 g \mu_{B} B_{z} S \frac{R}{M} .
$$

Zeeman energy increases linearly with the magnetic field. Activation energy is determined by the energy barrier between stable and metastable states (see Fig. 1), and for nanomagnets it may be found as

$$
E_{a}=D_{a} S^{2}-g \mu_{B} B_{z} S+\frac{g^{2}}{4 D_{a}} \mu_{B}^{2} B_{z}^{2}
$$

the activation energy is provided in temperature units. The dependence of Zeeman and activation energies on the applied magnetic field is presented in Fig. 5 for $\mathrm{Mn}_{12}$ acetate. As we can see, the activation energy decreases with the magnetic field and becomes zero at $B_{z} \approx 10 \mathrm{~T}$; at higher fields the potential barrier disappears, and the transition from state $S_{z}=-10$ to state $S_{z}=10$ is not hindered any more.

It is convenient to define a new dimensionless value $r \equiv$ $\rho / \rho_{0}=V_{0} / V$, which represents possible compression of the crystal in the process of magnetic detonation. Weak shock and detonation cause only elastic deformations, and the equation of state for the crystals of nanomagnets may be written as a combination of elastic and thermal components as $[20,24]$

$$
\frac{P}{\rho_{0}}=\frac{c_{0}^{2}}{n}\left(r^{n}-1\right)+\frac{R A \Gamma k_{B} T^{\alpha+1} r}{M(\alpha+1) \Theta_{D}^{\alpha}},
$$

where $M$ is the molecular mass with $M=1868 \mathrm{~g} / \mathrm{mol}$ for $\mathrm{Mn}_{12}$ acetate (see Ref. [3]). Like in Ref. [20], we take $n=4$, the Gruneisen coefficient $\Gamma=2$, the problem dimension $\alpha=$ 3 , and the Debye temperature $\Theta_{D}=38 \mathrm{~K}$. The coefficient $A=12 \pi^{4} / 5$ corresponds to the Debye crystal lattice, and $c_{0} \approx$ $2000 \mathrm{~m} / \mathrm{s}$ is the sound speed in the crystal. The thermodynamic energy per mass unit of the crystal is given by [20,24]

$$
\varepsilon=\frac{c_{0}^{2}}{n}\left(\frac{r^{n-1}-1}{n-1}+\frac{1}{r}-1\right)+\frac{R A T^{\alpha+1}}{M(\alpha+1) \Theta_{D}^{\alpha}} .
$$

Equations (9)-(11) provide a complete system for describing magnetic detonation parameters in crystals of nanomagnets with thermal conduction taken into account. 


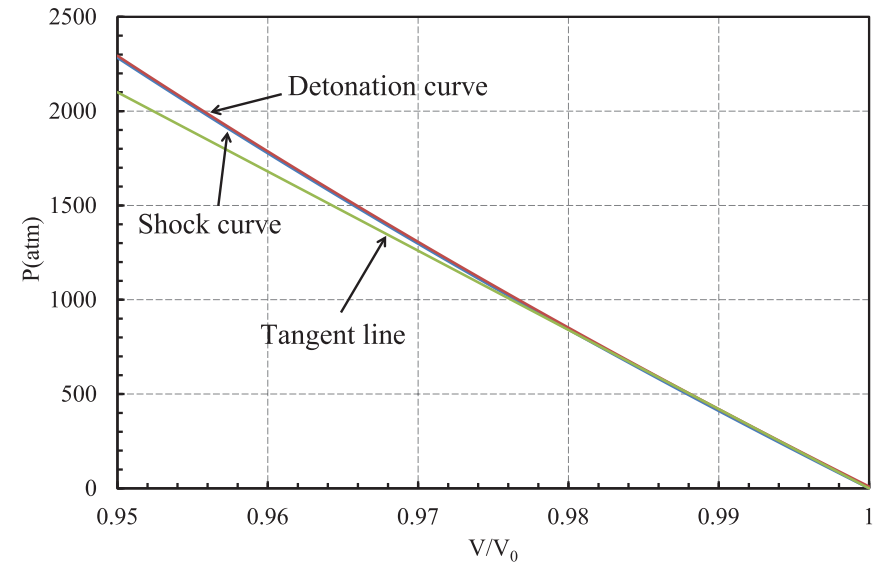

FIG. 6. (Color online) The pressure-volume diagram for the shock (blue) and magnetic detonation (red) in a $\mathrm{Mn}_{12}$ acetate crystal. The green line represents the tangent line from the initial state to the CJ regime; the magnetic field is $B_{z}=4 \mathrm{~T}$.

The solution to Eqs. (9)-(11) is presented in Fig. 6 in terms of the pressure-volume diagram for a magnetic detonation structure similar to that in Fig. 3. We can see immediately that since magnetic detonation is extremely weak, the shock curve (blue) and the detonation curve (red) almost coincide; as in Fig. 3, the tangent line from the initial state to the CJ detonation regime is shown by green. In the case of zero viscosity, pressure at the tangent line (labeled $t$ ) follows from Eqs. (9) and (10) as

$$
P_{t}=P_{0}+\rho_{0} D^{2}(1-1 / r) .
$$

In order to make the difference between the shock and detonation visible, we modify the pressure-volume diagram by extracting pressure at the tangent line, Eq. (17), i.e., by plotting $P-P_{t}$ versus $V$ (see Fig. 7). Within such a representation, the tangent line (by definition) becomes simply the zero line, while the shock and the detonation states may be easily distinguished and are represented by two curves resembling parabola pieces.

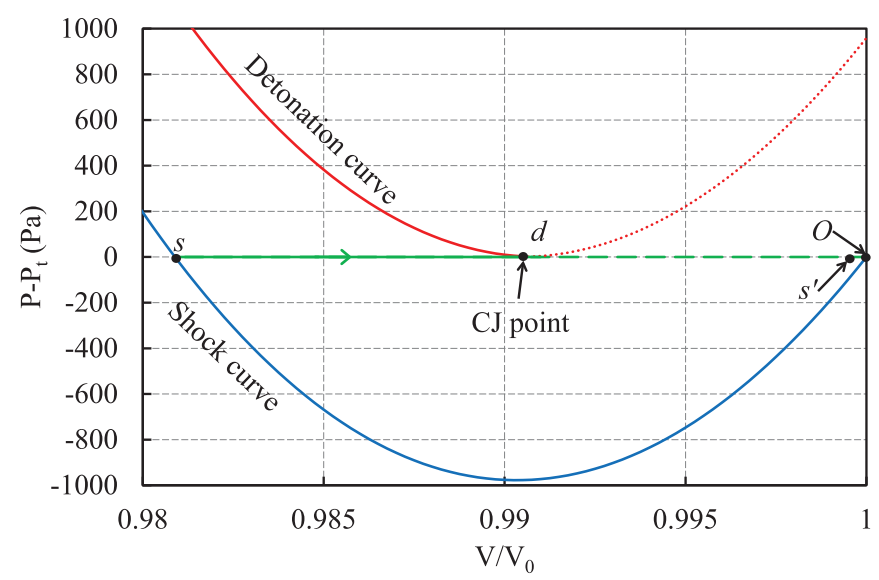

FIG. 7. (Color online) The modified pressure-volume diagram for $P-P_{t}$ for the shock (blue) and magnetic detonation (red) in a $\mathrm{Mn}_{12}$ acetate crystal. The reference pressure $P_{t}$ of the green line corresponds to the tangent line equation (17) from the initial state to the CJ regime; the magnetic field is $B_{z}=4 \mathrm{~T}$.

\section{B. The analytical theory for the key magnetic detonation parameters}

As we can see in Fig. 7, the limit of weak compression $(r-1) \ll 1$ holds with very good accuracy for magnetic detonations and hence allows us to develop the analytical theory of the process. The system (9)-(11) may be reduced to a single equation, which is equivalent to the Hugoniot relation [22] with thermal conduction taken into account:

$$
\varepsilon-\varepsilon_{0}-\frac{1}{2} \frac{r-1}{r} \frac{P_{0}+P}{\rho_{0}}+Q(a-1)=\frac{\kappa}{\rho_{0} D} \frac{d T}{d z} .
$$

By combining the equations of state for the crystals of nanomagnets, Eqs. (15) and (16), we get rid of the thermal terms and obtain the expression for thermodynamic energy as a function of only $P$ and $r$,

$$
\begin{aligned}
\varepsilon= & \frac{1}{r \Gamma} \frac{P}{\rho_{0}} \\
& +\frac{c_{0}^{2}}{n r}\left[\left(r^{n}-1\right)\left(\frac{1}{n-1}-\frac{1}{\Gamma}\right)-\frac{n}{n-1}(r-1)\right] .
\end{aligned}
$$

Then substituting Eq. (19) into the Hugoniot relation (18), we obtain pressure as

$$
\begin{aligned}
\frac{P}{\rho_{0}}= & \frac{2 \Gamma}{2-\Gamma(1-r)}\{r Q(1-a) \\
& +\left(\frac{r-1}{2} \Gamma+r\right) \varepsilon_{0}+r \frac{\kappa}{\rho_{0} D} \frac{d T}{d z} \\
& \left.+\frac{c_{0}^{2}}{n}\left[\frac{n}{n-1}(r-1)-\left(r^{n}-1\right)\left(\frac{1}{n-1}-\frac{1}{\Gamma}\right)\right]\right\} .
\end{aligned}
$$

In a similar way, by substituting Eqs. (15) and (16) into Eq. (18) we find temperature

$$
\begin{aligned}
T^{\alpha+1}= & \frac{2(\alpha+1) M \Theta_{D}^{\alpha}}{R A[2-\Gamma(1-r)]}\{Q(1-a) \\
& +\frac{c_{0}^{2}}{n r}\left[\frac{n}{n-1}(r-1)-\left(\frac{1}{n-1}-\frac{r-1}{2}\right)\right] \\
& \left.+\left(\frac{r-1}{2 r} \Gamma+1\right) \varepsilon_{0}+\frac{\kappa}{\rho_{0} D} \frac{d T}{d z}\right\} .
\end{aligned}
$$

Now we take into account the fact that density variations in the magnetic detonation are small by introducing a small value $\delta=r-1=\rho / \rho_{0}-1 \ll 1$. We can also neglect the initial thermodynamic energy since $\varepsilon_{0} / Q \sim 10^{-6}$. First, we analyze the detonation products, $z \rightarrow \infty$, for which the transformation process is completed, $d T / d z \rightarrow 0$. Expanding Eq. (20) in powers of $\delta$ and taking $a=0$, we obtain pressure in the detonation products as

$$
\begin{aligned}
\frac{P_{d}}{\rho_{0}}= & \Gamma Q+\left[c_{0}^{2}+\Gamma\left(\frac{\Gamma}{2}+1\right)\right] \delta_{d} \\
& +\frac{1}{2}\left[\frac{\Gamma^{2}}{2} Q\left(\frac{\Gamma}{2}+1\right)+c_{0}^{2}(n-1)\right] \delta_{d}^{2} .
\end{aligned}
$$

The scaled density deviation $\delta_{d}$ corresponding to the $\mathrm{CJ}$ point of the detonation products in Fig. 7 is unknown so far, and we 
have to find it. At the CJ point the tangent line touches the detonation curve, which implies

$$
\begin{gathered}
P_{t}=P_{d}, \\
\frac{\partial P_{t}}{\partial r}=\frac{\partial P_{d}}{\partial r} .
\end{gathered}
$$

For the small compression case $\delta \ll 1$ we rewrite Eq. (17) as

$$
\frac{P_{t}-P_{0}}{\rho_{0}}=D^{2} \delta(1-\delta) \text {. }
$$

Substituting Eqs. (22) and (25) into Eqs. (23) and (24), we find

$$
\begin{aligned}
D^{2} \delta_{d}\left(1-\delta_{d}\right)= & \Gamma Q+\left[c_{0}^{2}+\Gamma Q\left(\frac{\Gamma}{2}+1\right)\right] \delta_{d} \\
& +\frac{1}{2}\left[\frac{\Gamma^{2}}{2} Q\left(\frac{\Gamma}{2}+1\right)+c_{0}^{2}(n-1)\right] \delta_{d}^{2}, \\
D^{2}\left(1-2 \delta_{d}\right)= & c_{0}^{2}+\Gamma Q\left(\frac{\Gamma}{2}+1\right) \\
& +\left[\frac{\Gamma^{2}}{2} Q\left(\frac{\Gamma}{2}+1\right)+c_{0}^{2}(n-1)\right] \delta_{d} .
\end{aligned}
$$

Then, eliminating $D^{2}$ from Eqs. (26) and (27) and keeping terms as small as $\propto \delta_{d}^{2}$, we obtain

$$
\begin{aligned}
& \Gamma Q\left(1-2 \Gamma \delta_{d}\right) \\
& \quad=\delta_{d}^{2}\left[\Gamma Q\left(1+\frac{\Gamma}{2}\right)\left(1+\frac{\Gamma}{4}\right)+\frac{1}{2} c_{0}^{2}(n+1)\right]
\end{aligned}
$$

We may also use the condition $4 \Gamma^{2} Q \ll \delta_{d}(n+1) c_{0}^{2}$, which is justified below, to simplify Eq. (28), and we find the density deviations in the detonation products as

$$
\delta_{d}=\frac{1}{c_{0}} \sqrt{\frac{2 \Gamma Q}{n+1}} .
$$

As an example, for the external magnetic field of $B_{z}=4 \mathrm{~T}$ we obtain density deviations from the initial value as small as $\delta_{d} \approx 9.6 \times 10^{-3} \ll 1$. The temperature of the detonation products may be calculated with the accuracy of the first-order terms in $Q \delta_{d}$ as

$$
\begin{aligned}
T_{d}^{\alpha+1} & =\frac{(\alpha+1) M \Theta_{D}^{\alpha}}{R A}\left[Q\left(1+\frac{\Gamma}{2} \delta_{d}\right)+c_{0}^{2} \frac{n+1}{12} \delta_{d}^{3}\right] \\
& =\frac{(\alpha+1) Q M \Theta_{D}^{\alpha}}{R A}\left(1+\frac{7 \Gamma}{6 c_{0}} \sqrt{\frac{\Gamma Q}{2(n+1)}}\right) .
\end{aligned}
$$

Figure 8 shows the density, temperature, and pressure of the detonation products versus the applied magnetic field as found in the numerical solution and predicted by the analytical formulas (22), (29), and (30); we observe excellent agreement of the theory with the numerical solution. We also point out that the magnetic detonation speed $D^{2}$ may be obtained from Eq. (27) up to the leading terms in $\delta_{d}$ as

$$
\begin{aligned}
D^{2}= & \frac{1}{1-2 \delta_{d}}\left\{c_{0}^{2}+\Gamma Q\left(\frac{\Gamma}{2}+1\right)\right. \\
& \left.+\left[\frac{\Gamma^{2}}{2} Q\left(\frac{\Gamma}{2}+1\right)+c_{0}^{2}(n-1)\right] \delta_{d}\right\} .
\end{aligned}
$$
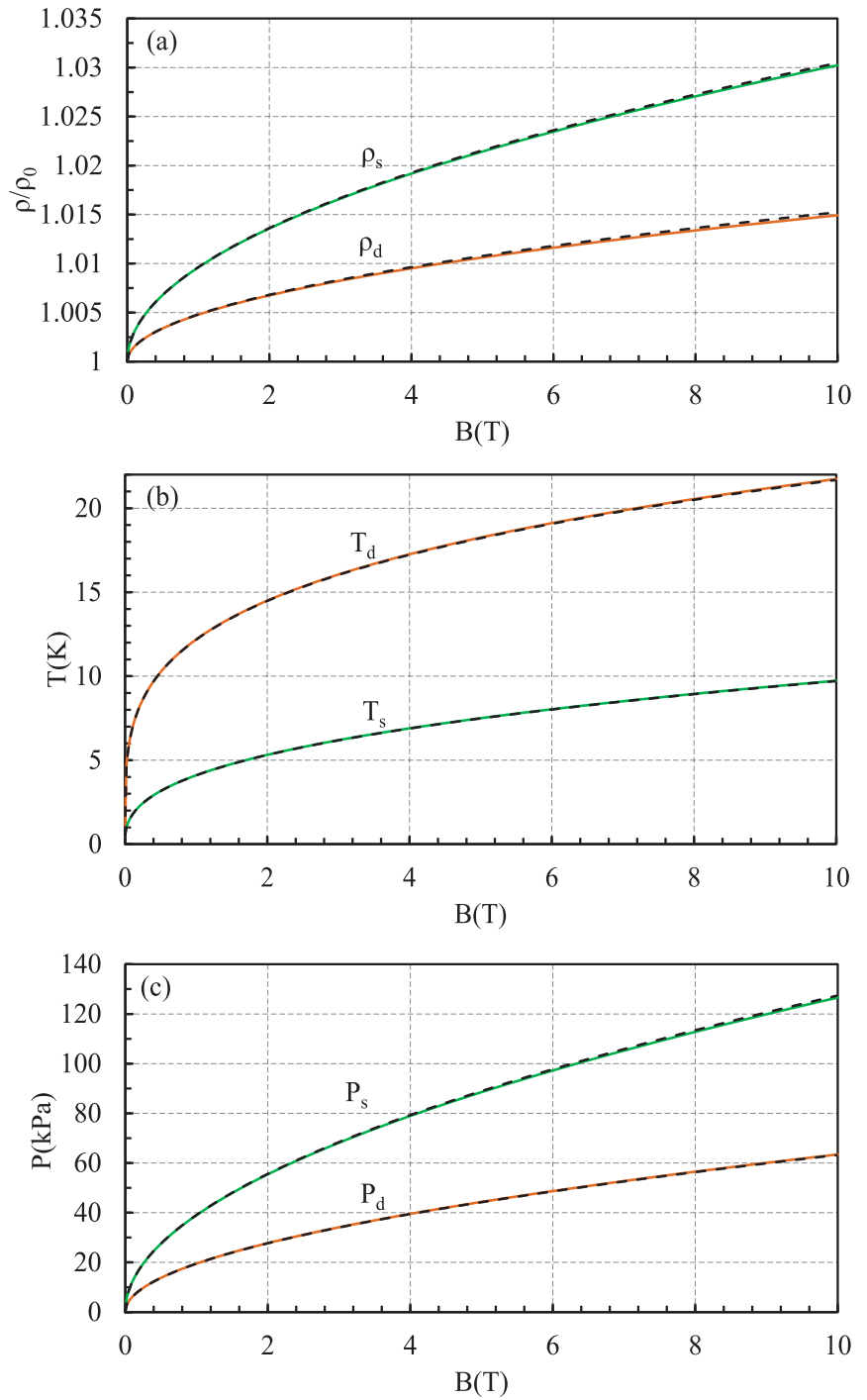

FIG. 8. (Color online) (a) Density, (b) temperature, and (c) pressure at the leading shock and in the magnetic detonation products vs the external magnetic field. Solid lines correspond to the numerical solution, and the dashed lines represent the analytical theory.

Keeping the leading terms, we can rewrite Eq. (31) as

$$
D \approx c_{0}+\frac{\Gamma Q}{2 c_{0}^{2}}\left(\frac{\Gamma}{2}+1\right)+\frac{\delta_{d}}{2}\left[\frac{\Gamma Q}{2 c_{0}^{2}}\left(\frac{\Gamma}{2}+1\right)^{2}+(n+1)\right] .
$$

Substituting Eq. (29) in Eq. (32) and taking into account $\Gamma Q / c_{0}^{2} \ll 1$, we find the magnetic detonation speed

$$
D \approx c_{0}+\sqrt{\frac{n+1}{2} \Gamma Q} .
$$

Thus, in agreement with Ref. [20], the magnetic detonation speed only slightly exceeds the sound speed in the crystals.

It is also useful to find the thermodynamic parameters of the crystal just behind the shock, $a=1$, neglecting thermal conduction, i.e., treating the shock as a discontinuity, similar to what was done in Ref. [20]. We will show in the next section that the same values for temperature, pressure, and 
density hold at the isothermal discontinuity while taking into account thermal conduction. So we are looking for the density deviations $\delta_{s}$ corresponding to the crystal just behind an infinitely thin shock. Equation (20) yields up to the second-order terms in $\delta$,

$$
\frac{P_{s}}{\rho_{0}}=\delta c_{0}^{2}\left[1+\frac{1}{2} \delta(n-1)\right] .
$$

The tangent line intersects the shock curve at point $s$, which implies $P_{s}=P_{t}$ or

$$
D^{2} \delta_{s}\left(1-\delta_{s}\right)=\delta_{s} c_{0}^{2}\left[1+\frac{1}{2} \delta_{s}(n-1)\right] .
$$

Taking into account the result for the magnetic detonation speed, Eq. (31), we obtain the equation for $\delta_{s}$ :

$$
\begin{aligned}
& \left(1-\delta_{s}\right)\left\{c_{0}^{2}+\left[\frac{\Gamma^{2}}{2} Q\left(\frac{\Gamma}{2}+1\right)+c_{0}^{2}(n-1)\right] \delta_{d}\right. \\
& \left.\quad+\Gamma Q\left(\frac{\Gamma}{2}+1\right)\right\}=\left(1-2 \delta_{d}\right) c_{0}^{2}\left[1+\frac{1}{2} \delta_{s}(n-1)\right],
\end{aligned}
$$

which may be solved with the accuracy of $\Gamma Q \ll c_{0}^{2} \delta$ as

$$
\delta_{s} \approx 2 \delta_{d} .
$$

It is noted that density deviation just behind the discontinuous shock is about two times larger than in the detonation products, which may be seen already in Fig. 7. Then we find temperature on the shock similar to Eq. (30),

$$
\begin{aligned}
T_{s}^{\alpha+1} & =\frac{(\alpha+1) M \Theta_{D}^{\alpha}}{R A} \frac{n+1}{12} c_{0} \delta_{s}^{3} \\
& \approx \frac{4(\alpha+1) \Gamma Q M \Theta_{D}^{\alpha}}{3 c_{0} R A} \sqrt{\frac{2 \Gamma Q}{n+1}} .
\end{aligned}
$$

Density, temperature, and pressure at the leading shock in magnetic detonation are shown in Fig. 8; we again observe a very good agreement of the analytical theory and the numerical solution.

\section{Isothermal discontinuity in magnetic detonation}

In this section we obtain the magnetic detonation structure in the presence of thermal conduction. By using Eqs. (15) and (17) we find temperature as a function of scaled density $r$ inside the magnetic detonation front

$$
T^{\alpha+1}=\frac{(\alpha+1) M \Theta_{D}^{\alpha}}{\Gamma R A r}\left[D^{2}\left(1-\frac{1}{r}\right)-\frac{c_{0}^{2}}{n}\left(r^{n}-1\right)\right] .
$$

By plotting temperature $T$ according to Eq. (39) from the initial point $O$ to the shock point $s$, we observe nonmonotonic temperature variations (see Fig. 9), with the maximum value attained at the detonation point $d$. In that case, assuming hypothetic temperature variations along the red curve (solid and dashed) in Fig. 9 from $O$ to $d$ in the whole detonation front, one moves first from $O$ to $s^{\prime}$, then passes $d$ to the shock point $s$, and then returns back from $s$ to the final state $d$. Along such a pass we obtain the region with temperature decrease $d T / d x<0$ on the way from $d$ to the shock point $s$. However, the left-hand side of Eq. (18) becomes zero only at the boundaries of the detonation wave $z \rightarrow \pm \infty$; that is, it has to be either positive or negative in the transition

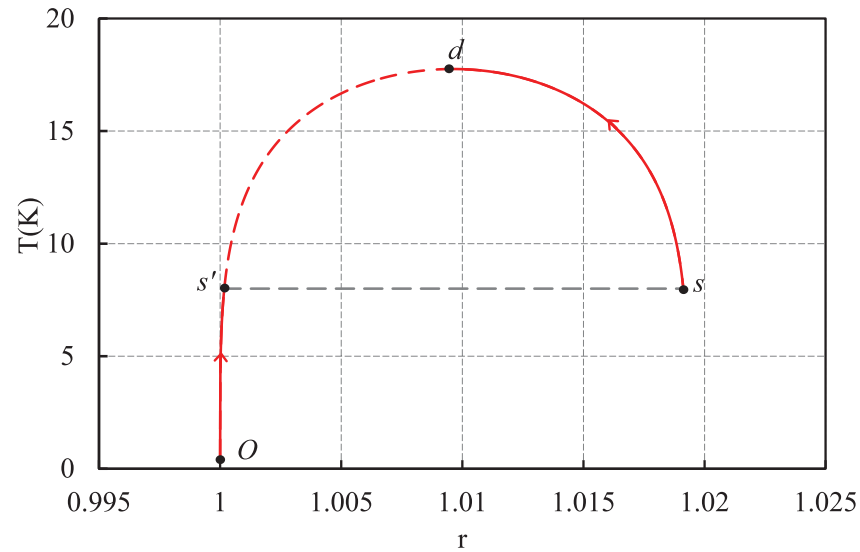

FIG. 9. (Color online) Isothermal discontinuity diagram. Temperature dependence on $V / V_{0}$ according to Eq. (39). Starting from the initial point $O$, all parameters vary continuously to $s^{\prime}$. Then pressure and density experience isothermal discontinuity from point $s^{\prime}$ to point $s$ (black dashed line). The red dashed line corresponds to the nonphysical region in the shock phase. From point $s$, the system relaxes to the final state $d$ in the process of spin flipping (magnetic detonation). Crystal compression at point $s^{\prime}$ is $V / V_{0}=0.99988$, corresponding to $r \equiv \rho / \rho_{0}=1.00012$.

zone from 0 to $d$ (see Refs. [22,24]). Since detonation definitely leads to temperature increase from the initial state, we have the condition $d T / d x>0$ satisfied everywhere in the magnetic detonation front, so that the hypothetical region with $d T / d x<0$ is not physical and should be avoided. Then, variations of all values of magnetic detonation in Fig. 9 have to correspond to continuous movement from the initial point $O$ to point $s^{\prime}$, which is followed by a jump to the shock point $s$, with subsequent continuous evolution to the final detonation point $d$. At the same time, because of thermal conduction in Eqs. (9)-(11), temperature has to be continuous in magnetic detonation including the shock wave. For that reason, instead of a traditional shock, we obtain an isothermal discontinuity in the magnetic detonation in the case of negligible volume viscosity when the shock structure is supported by only thermal conduction. An important point is that compression of the crystal in the point $s^{\prime}$ is quite small, $r=1.00012$, so that the isothermal discontinuity resembles a shock for density and pressure. The visual difference is found only for temperature variations. Although interesting from a theoretical point of view, such a tiny jump is hardly possible to observe experimentally. Furthermore, we will show in the next section that volume viscosity influences the magnetic detonation structure much more strongly, making temperature, density, and pressure profiles smooth all over the front.

The internal structure of magnetic detonation can be obtained by integrating the equation for kinetics of spin relaxation [14], which strongly resembles the Arrhenius law of chemical kinetics (7),

$$
\frac{\partial a}{\partial t}=-\frac{a}{\tau} \exp \left(-E_{a} / T\right)
$$

If we adopt the reference frame of the stationary magnetic detonation front, Eq. (40) can be written as

$$
u \frac{\partial a}{\partial x}=-\frac{a}{\tau} \exp \left(-E_{a} / T\right) .
$$




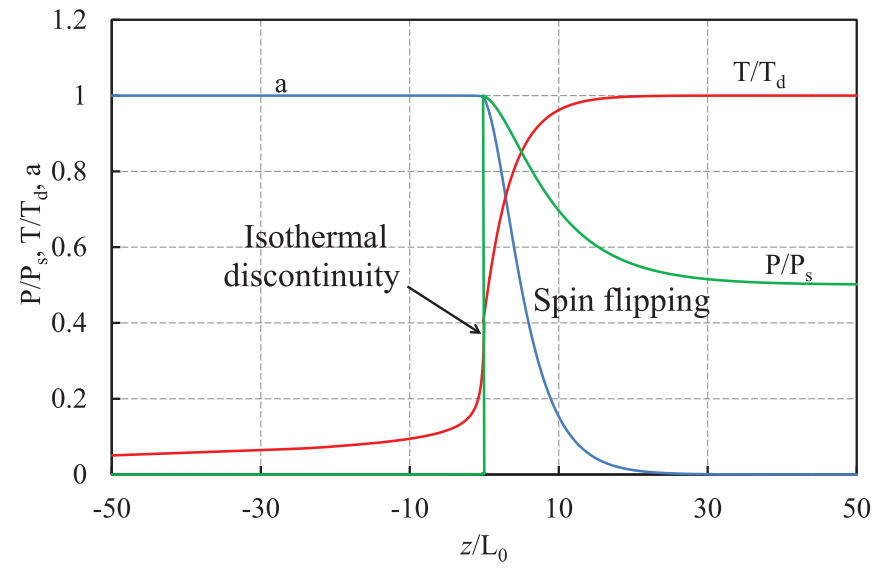

FIG. 10. (Color online) Magnetic detonation structure in $\mathrm{Mn}_{12}$ acetate crystals in the external magnetic field $B_{z}=4 \mathrm{~T}$. The plots are scaled by the temperature of the detonation products $T_{d}=17.25 \mathrm{~K}$ and the shock pressure $P_{s} \approx 80 \mathrm{kPa}$. Other parameters are specified in the text.

By using Eq. (39), we solve Eq. (41) numerically, going from the initial point $O$ to the point of isothermal discontinuity $s^{\prime}$. After that, new values for density and pressure at the point $s$ are calculated according to the jump conditions, with temperature and fraction of metastable molecules $a$ kept constant. Then a numerical calculation is performed from point $s$ to the final detonation state $d$. The result of the numerical solution is presented in Fig. 10 for the $\mathrm{Mn}_{12}$ acetate crystals with the characteristic thickness of the detonation front $L_{0} \equiv c_{0} \tau \approx 0.2 \mathrm{~mm}$ employed as the length scale. In Fig. 10 for illustrative purposes we plot the numerical solution for the thermal conduction coefficient $\kappa=10^{4} \mathrm{~m}^{2} / \mathrm{s}$, which is about nine orders larger than the commonly accepted value $\kappa \approx 2 \times 10^{-5} \mathrm{~m}^{2} / \mathrm{s}$ (e.g., see Refs. [14,17]). For realistic values of $\kappa$ the shock part of the front is negligible compared to the spin-flipping part and can hardly be distinguished from the case of zero thermal conduction. Still, even in that case of high $\kappa$, the thickness of the heating region is much smaller than that of the region of spin flipping, and the fraction of the molecules in the metastable state is close to unity at the isothermal discontinuity, $a=0.993$.

Thus, as an intermediate conclusion, thermal conduction influences only the temperature profile in magnetic detonation, with a minor effect on density and pressure and with negligible modifications of the total front thickness. Much more dramatic modifications of the magnetic detonation structure are expected because of volume viscosity, as shown in the next section.

\section{DETONATION FRONT WITH VISCOSITY}

By taking into account volume viscosity $\eta$, we rewrite the equations of mass, momentum, and energy conservation for magnetic detonation as

$$
\begin{gathered}
\rho_{0} D=\rho u, \\
P_{0}+\rho_{0} D^{2}=P+\rho u^{2}-\eta \frac{d u}{d x},
\end{gathered}
$$

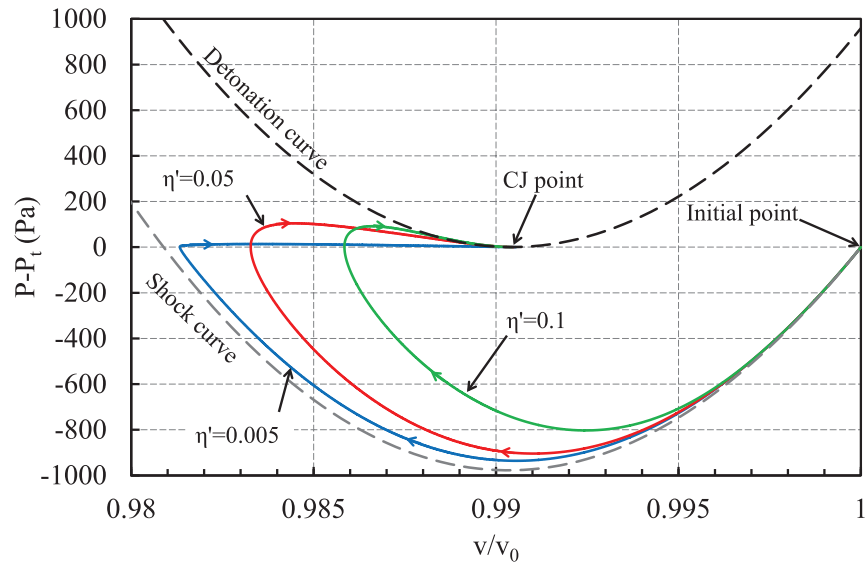

FIG. 11. (Color online) Pressure-volume diagram for different values of the scaled viscosity $\eta^{\prime}=0.005,0.05,0.1$ in the external magnetic field $B_{z}=4 \mathrm{~T}$.

$$
\begin{aligned}
\rho_{0} D & \left(\epsilon_{0}+\frac{P_{0}}{\rho_{0}}+\frac{D^{2}}{2}\right) \\
& =\rho u\left(\epsilon+\frac{P}{\rho}+\frac{u^{2}}{2}\right)-\kappa \frac{d T}{d x}-\eta u \frac{d u}{d x} .
\end{aligned}
$$

It is convenient to characterize the role of viscosity by the dimensionless parameter

$$
\eta^{\prime} \equiv \frac{\eta}{\rho_{0} c_{0} L_{0}}=\frac{\eta}{\rho_{0} c_{0}^{2} \tau} .
$$

This dimensionless viscosity plays a role conceptually similar to that of the inverse Reynolds number in fluid mechanics [22]. In particular, the parameter values $\eta^{\prime}=0.005,0.05,0.1$ employed below correspond to the domain of Reynolds numbers $\operatorname{Re}=10-200$. At such values of the Reynolds number gas and fluid flows are typically laminar; the negligible role of viscosity is qualitatively indicated by transition to turbulence, which usually happens at larger values, $\operatorname{Re} \sim 10^{3}$ and above. To the best of our knowledge, there have been no works, either experimental or theoretical, investigating volume viscosity in crystals of nanomagnets, and therefore we will take $\eta^{\prime}$ as a free parameter.

We stress that even small values of volume viscosity $\eta^{\prime} \sim$ 0.01 lead to considerable changes in the magnetic detonation structure, as shown in Figs. 11-14. Even in the pressurevolume diagram in Fig. 11 (plotted, as before, for $P-P_{t}$ by extracting the $\mathrm{CJ}$ tangent line for illustrative purposes), we observe that all discontinuous jumps of the previous model [20] are replaced by continuous transition lines from the initial point to the CJ detonation products. For very small viscosity, $\eta^{\prime}=0.005$, the transition line (blue), although continuous, goes pretty close to the Hugoniot curve for the shock and then to the tangent line for the spin-flipping process. In a similar way, in Fig. 13 the plot for $\eta^{\prime}=0.005$ demonstrates a quite abrupt initial pressure increase, almost a jump, which strongly resembles a discontinuous shock wave; this pressure increase is followed by pressure relaxation to a smaller value in the process of spin flipping with Zeeman energy release. As we take larger values of volume viscosity, $\eta^{\prime}=0.05,0.1$, deviation of the pressure-volume plots from the discontinuous 


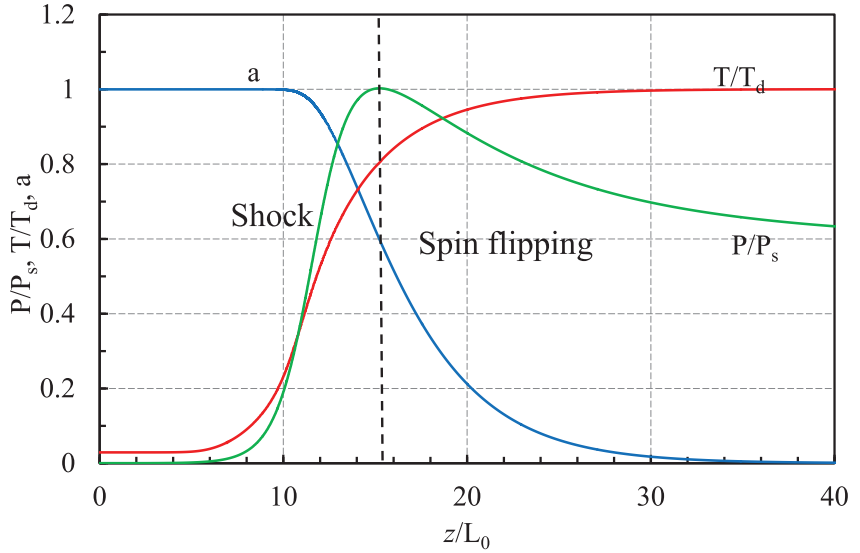

FIG. 12. (Color online) Profiles of scaled pressure, temperature, and fraction of nanomagnets in the metastable state for magnetic detonation for scaled viscosity $\eta^{\prime}=0.05$ in the external magnetic field $B_{z}=4 \mathrm{~T}$ corresponding to the blue curve in Fig. 11 .

shock model becomes much more pronounced (Fig. 11), and we find pressure and density in the shock wave decreasing compared to those in the discontinuous case (Fig. 13). In fact, the very definition of a shock as a part of the magnetic detonation front becomes ambiguous when volume viscosity is taken into account. To avoid the ambiguity, we notice that within the model of a discontinuous shock, the pressure maximum is attained at the shock front, and then pressure goes down as the spin flipping starts. In the same way it seems natural to treat the point of maximal pressure in Figs. 12 and 13 as the back side of the shock region. In Fig. 13 we placed all three plots by choosing $z=0$ as the position of the pressure maximum and hence the back side of the shocks. Then the regions corresponding to $z<0$ belong to the shocks smoothed by viscosity, while the domain of $z>0$ may be treated roughly as the regions of spin flipping with Zeeman energy release. Of course, such separation is qualitative rather than quantitative because in the presence of considerable volume viscosity spin flipping starts already in the shock (see Fig. 12,

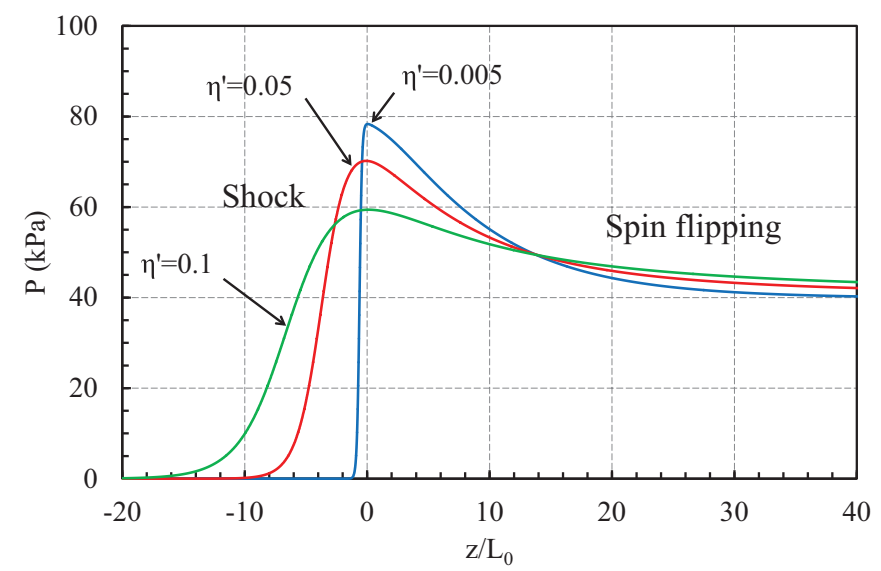

FIG. 13. (Color online) Pressure profiles in magnetic detonation for different values of the scaled viscosity $\eta^{\prime}=0.005,0.05,0.1$ in the external magnetic field $B_{z}=4 \mathrm{~T}$. Pressure maxima are placed at the position $z=0$, which may be defined as the back side of the shock region in the detonation.

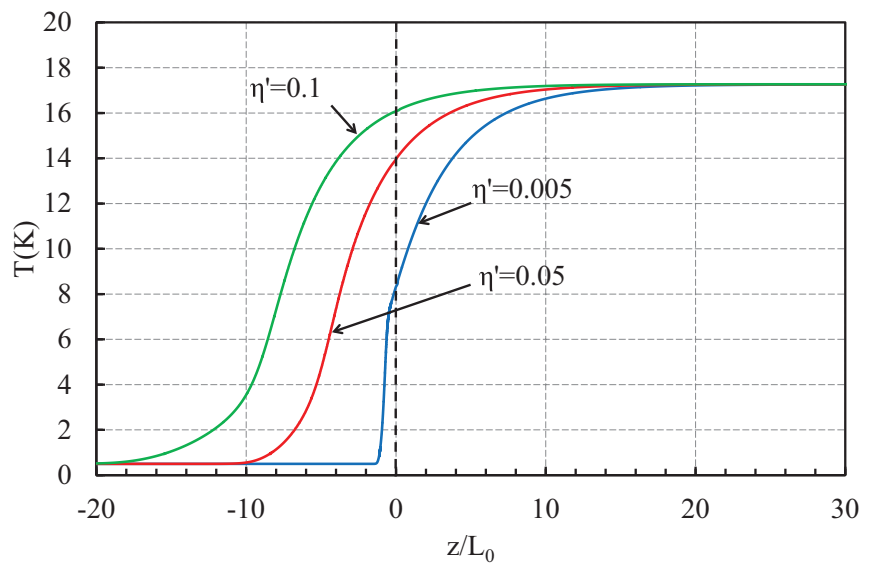

FIG. 14. (Color online) Temperature profiles in magnetic detonation for different values of the scaled viscosity $\eta^{\prime}=0.005,0.05,0.1$ in the external magnetic field $B_{z}=4 \mathrm{~T}$. As in Fig. 13, position $z=0$ corresponds to the maximum pressure for each profile treated as the back side of the shock wave.

corresponding to $\left.\eta^{\prime}=0.05, B_{z}=4 \mathrm{~T}\right)$. In Fig. 12 the fraction of nanomagnets in the metastable state is about $a \approx 0.6$ at the point of pressure maximum. Figure 14 compares temperature profiles for the scaled viscosity $\eta^{\prime}=0.005,0.05,0.1$ with the respective pressure maxima still placed at $z=0$. As we can see, a larger volume viscosity strongly increases temperature at the shock; in the case of $\eta^{\prime}=0.1$, the temperature at $z=0$ is only $5 \%$ smaller than the final detonation temperature, i.e., $\sim 0.95 T_{d}$. The temperature increase strongly reduces the size of the "pure" spin-flipping region; still, as we can see from Fig. 14, it is accompanied by a strong increase in the width of the shock wave, so that the total width of the magnetic detonation front does not change much, staying about $\sim 10 L_{0}$ for all three cases, i.e., about 1-2 mm in dimensional units.

We point out that the experimentally employed sample sizes for the crystals of nanomagnets are also about $2 \mathrm{~mm}$ (e.g., see Refs. [3-6,19]). As a result, it is rather difficult to observe steady, well-developed magnetic detonation in common experimental conditions since such observations require samples much larger than the detonation front thickness. Instead, we suggest that most of the experimental points reported in Ref. [19] for ultrafast magnetic avalanches correspond to magnetic detonation in the process of development, which is also indicated by the average avalanche speed in the samples being noticeably below the sound speed. Then, in order to observe a well-developed magnetic detonation one has to perform experiments with much bigger crystals of nanomagnets, simultaneously reducing the detonation front thickness as much as possible. In the case of relatively small viscosity, e.g., $\eta^{\prime}=0.005$, the detonation front thickness may be reduced by decreasing the scaled activation energy of spin flipping $E_{a} / T_{s}$. This, in turn, may be achieved by increasing the external magnetic field $B_{z}$ or by adding a transverse-magnetic-field component $B_{x}$, as we show below. In some respects the transverse field also mimics the presence of the transverse-magnetic anisotropy, which may lead to splitting of spin states even at zero magnetic field and, consequently, to changing the activation energy. Moreover, if the transverse anisotropy is present and both uniaxial and 


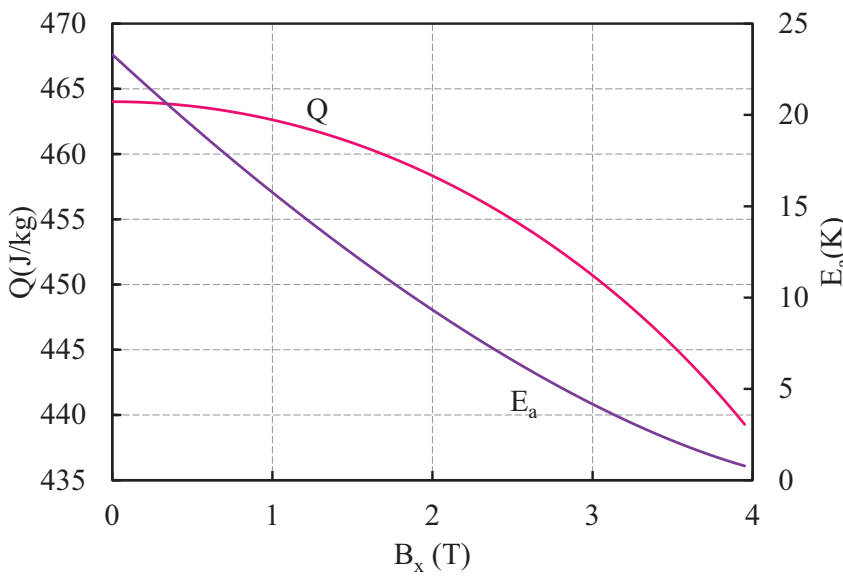

FIG. 15. (Color online) Zeeman energy $Q$ and activation energy $E_{a}$ vs the transverse magnetic field $B_{x}$ at $B_{z}=4 \mathrm{~T}$.

transverse components of magnetic field are applied, one can expect that the presence of the diabolical points should also manifest in the behavior of the fronts [26]. Another important feature of the experimental observations of Ref. [19] is that the ultrafast avalanches were obtained for the magnetic field close to the quantum resonance values of the nanomagnets. Quantum resonances lead to a strong decrease in the factor $\tau$ in the kinetic equation of spin flipping, Eq. (40), and hence of the characteristic width of the magnetic detonation front $\propto L_{0}=c_{0} \tau$, which may allow experimental observation of magnetic detonation. In addition, successful experiments on magnetic detonation should provide as fine a placement of Hall sensors as possible in order to get sufficient resolution to see the detonation front structure. Another possible way of spatially resolving the magnetic detonation front is magneto-optical imaging [27]. Still, prior to performing magnetic detonation experiments, the volume viscosity coefficient in the crystals of nanomagnets must be measured because this value influences the detonation front thickness quite strongly.

One of the efficient ways of reducing the thickness of the magnetic detonation front is to apply a transverse component of the magnetic field $B_{x}$ perpendicular to the easy axis of the crystal. By taking into account the transverse component, the Hamiltonian for the nanomagnet may be written as [17]

$$
\mathcal{H}=-D_{a} S_{z}^{2}-g \mu_{B}\left(B_{z} S_{z}+B_{x} S_{x}\right) .
$$

Here we calculate the activation energy by using the quasiclassical approach from Ref. [17].

The respective dependences of the Zeeman energy $Q$ and the activation energy $E_{a}$ on the transverse magnetic field $B_{x}$ are demonstrated in Fig. 15 for fixed strength of the longitudinal component, $B_{z}=4 \mathrm{~T}$. As we can see, an increase in the transverse component $B_{x}$ leads to a considerable reduction of the activation energy $E_{a}$, which is accompanied by minor modifications of the Zeeman energy $Q$. A strong reduction in the activation energy speeds up the process of spin flipping, which naturally implies a reduction of the detonation front thickness.

A reduction of the magnetic detonation thickness may be observed in Fig. 16 for pressure profiles plotted for different values of the transverse magnetic field $B_{x}$ for the cases of small
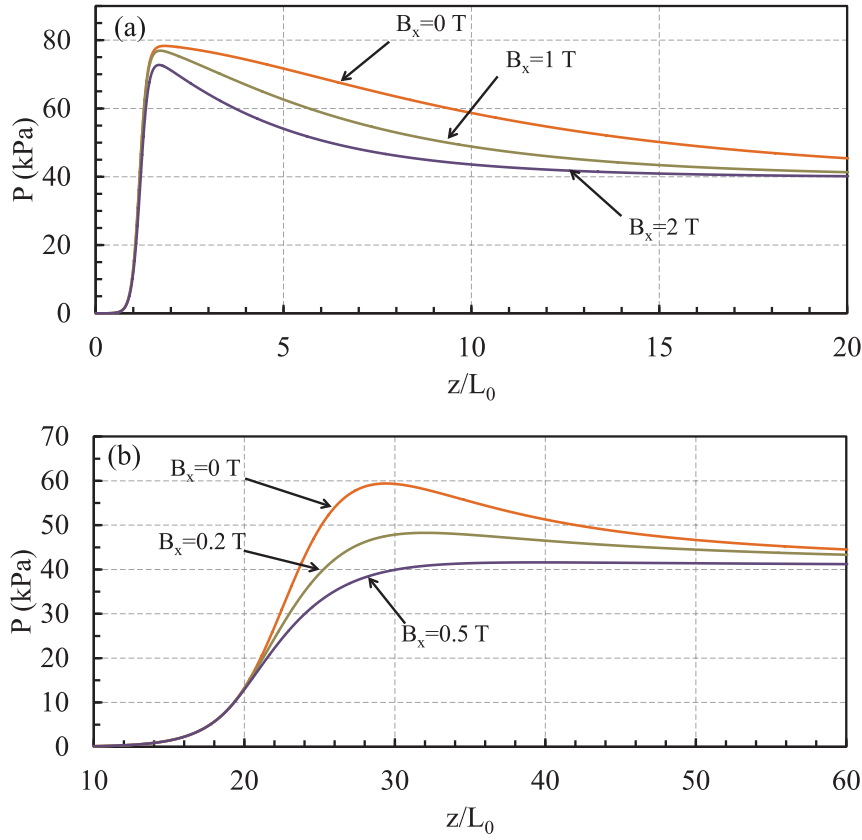

FIG. 16. (Color online) Pressure profiles in magnetic detonation for different values of the scaled viscosity (a) $\eta^{\prime}=0.005$ and (b) $\eta^{\prime}=0.1$ in the longitudinal magnetic field $B_{z}=4 \mathrm{~T}$ for different values of the transverse magnetic field $B_{x}$.

viscosity, $\eta^{\prime}=0.005$ [Fig. 16(a)], and large viscosity, $\eta^{\prime}=$ 0.1 [Fig. 16(b)]. In the case of small viscosity the transverse magnetic field does not modify the shock part of the magnetic detonation front and reduces slightly the peak pressure value. At the same time, by increasing the transverse field, e.g., from 0 to $2 \mathrm{~T}$, we may strongly reduce the size of the region of spin flipping with much faster relaxation to the final pressure value. In contrast, at a relatively high volume viscosity, $\eta^{\prime}=$ 0.1 , the increase in the transverse magnetic field affects the whole structure of the front. As we can see in Fig. 16(b), even a rather moderate increase in the transverse magnetic field from 0 to $0.5 \mathrm{~T}$ decreases the peak pressure value so much that the region of postshock spin flipping practically disappears, and almost the whole transformation process is accomplished within the leading shock. Such a structure is qualitatively different from that of the traditional combustion detonation described in Sec. II.

In order to understand the quantitative changes in the magnetic detonation thickness because of the applied transverse magnetic field, we have to introduce an accurate definition of the detonation thickness. Here we define the dimensionless front thickness $L_{t}$ (scaled by $L_{0}$ ) as the distance between two reference points corresponding to pressure deviation by $0.2\left(P_{d}-P_{0}\right)$ from the initial pressure $P_{0}$ and the final pressure $P_{d}$. Using such a definition, the dependence of the scaled magnetic detonation thickness $L_{t}$ on the transverse magnetic field $B_{x}$ is shown in Fig. 17 for different viscosity values $\eta^{\prime}$. We can see that, for each value of volume viscosity, the detonation front thickness may be reduced almost by an order of magnitude by increasing the transverse magnetic field. Another interesting effect observed in Fig. 17 is the abrupt decrease in the magnetic detonation thickness at a certain 


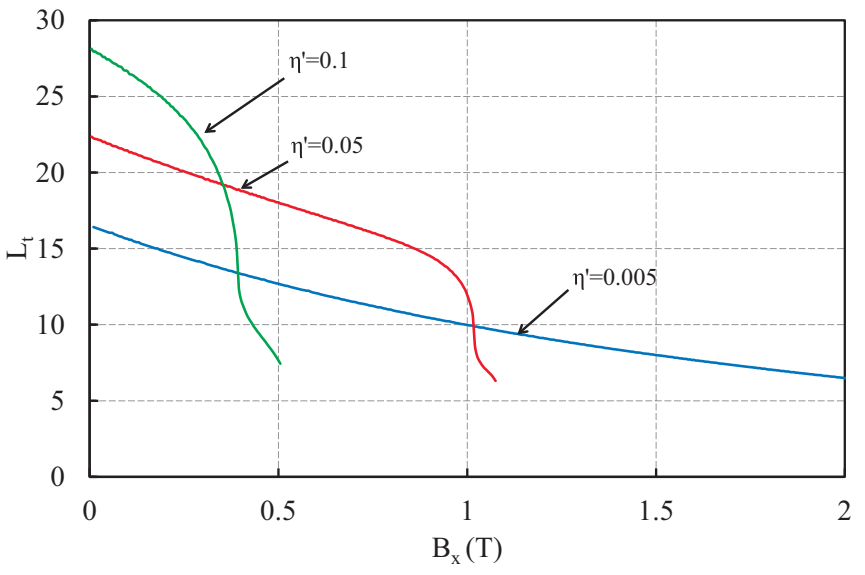

FIG. 17. (Color online) Scaled thickness of the magnetic detonation front $L_{t}$ vs the transverse magnetic field $B_{x}$ for different values of the scaled viscosity $\eta^{\prime}=0.005,0.05,0.1$ for the longitudinal magnetic field $B_{z}=4 \mathrm{~T}$.

critical transverse magnetic field (the value of the critical field depends on viscosity). For example, in the case of $\eta^{\prime}=0.05$ we observe a sudden drop in the detonation thickness at $B_{x} \approx 1 \mathrm{~T}$; for $\eta^{\prime}=0.1$ the drop happens at $B_{x} \approx 0.4 \mathrm{~T}$. This sudden drop in the magnetic detonation thickness indicates the instant when peak pressure in the detonation front becomes smaller than the value $0.2\left(P_{d}-P_{0}\right)$ employed to define $L_{t}$. In that case the region of postshock spin flipping effectively disappears, and the detonation thickness is determined by only the leading shock.

\section{SUMMARY}

In the present paper we have investigated the internal structure of magnetic detonation in crystals of nanomagnets.
Magnetic detonation is weak and propagates with speed only slightly exceeding the sound speed in the crystals. For that reason, in stark contrast to the usual combustion detonations, transport processes, thermal conduction and volume viscosity, play an important role in forming the magnetic detonation structure. We show that, in the case of negligible volume viscosity, thermal conduction produces isothermal discontinuity instead of the leading shock in magnetic detonation. In the isothermal discontinuity the temperature of the crystal is continuous, while density and pressure experience a jump.

Volume viscosity leads to much more dramatic changes in the magnetic detonation structure than in the model of Ref. [20] with neglected transport processes. In that case all the important thermodynamic parameters of the crystals acquire smooth profiles all over the magnetic detonation front including the leading shock. In addition, the very concept of the leading shock requires unambiguous definition; here we suggest specifying the back side of the shock as the position of the pressure maximum. As the relative role of volume viscosity increases, the leading shock becomes wider and may considerably exceed the zone of spin flipping by size. Still, the total size of a magnetic detonation front does not change much with variations in volume viscosity since the decrease of the spin-flipping region is compensated by the increase of the shock width. At the same time, the magnetic detonation thickness may be reduced by an order of magnitude by applying the transverse magnetic field to the crystals of nanomagnets, which is important for possible experimental observations of magnetic detonation.

\section{ACKNOWLEDGMENT}

This work has been supported by the Swedish Research Council (VR).
[1] J. Tejada, E. M. Chudnovsky, E. del Barco, J. M. Hernandez, and T. P. Spiller, Nanotechnology 12, 181 (2001).

[2] L. Bogani and W. Wernsdorfer, Nat. Mater. 7, 179 (2008).

[3] S. McHugh and M. P. Sarachik, Mod. Phys. Lett. B 25, 1795 (2011).

[4] Y. Suzuki, M. P. Sarachik, E. M. Chudnovsky, S. McHugh, R. Gonzalez-Rubio, N. Avraham, Y. Myasoedov, E. Zeldov, H. Shtrikman, N. E. Chakov, and G. Christou, Phys. Rev. Lett. 95, 147201 (2005).

[5] A. Hernández-Mínguez, J. M. Hernandez, F. Macià, A. GarcíaSantiago, J. Tejada, and P. V. Santos, Phys. Rev. Lett. 95, 217205 (2005).

[6] P. Subedi, S. Vélez, F. Macià, S. Li, M. P. Sarachik, J. Tejada, S. Mukherjee, G. Christou, and A. D. Kent, Phys. Rev. Lett. 110, 207203 (2013).

[7] R. Sessoli, D. Gatteschi, A. Caneschi, and M. A. Novak, Nature (London) 365, 141 (1993).

[8] J. Villain, F. Hartman-Boutron, R. Sessoli, and A. Rettori, Europhys. Lett. 27, 159 (1994).

[9] J. R. Friedman, M. P. Sarachik, J. Tejada, and R. Ziolo, Phys. Rev. Lett. 76, 3830 (1996).

[10] L. Thomas, F. Lionti, R. Ballou, D. Gatteschi, R. Sessoli, and B. Barbara, Nature (London) 383, 145 (1996).
[11] E. M. Chudnovsky and J. Tejada, Macroscopic Quantum Tunneling of the Magnetic Moment (Cambridge University Press, Cambridge, 1998).

[12] W. Wernsdorfer, Adv. Chem. Phys. 118, 99 (2001).

[13] D. Gatteschi and R. Sessoli, Angew. Chem. Int. Ed. 42, 268 (2003).

[14] D. A. Garanin and E. M. Chudnovsky, Phys. Rev. B 76, 054410 (2007).

[15] M. Modestov, V. Bychkov, and M. Marklund, Phys. Rev. B 83, 214417 (2011).

[16] S. McHugh, B. Wen, X. Ma, M. P. Sarachik, Y. Myasoedov, E. Zeldov, R. Bagai, and G. Christou, Phys. Rev. B 79, 174413 (2009).

[17] C. M. Dion, O. Jukimenko, M. Modestov, M. Marklund, and V. Bychkov, Phys. Rev. B 87, 014409 (2013).

[18] O. Jukimenko, C. M. Dion, M. Marklund, and V. Bychkov, Phys. Rev. Lett. 113, 217206 (2014).

[19] W. Decelle, J. Vanacken, V. V. Moshchalkov, J. Tejada, J. M. Hernández, and F. Macià, Phys. Rev. Lett. 102, 027203 (2009).

[20] M. Modestov, V. Bychkov, and M. Marklund, Phys. Rev. Lett. 107, 207208 (2011).

[21] C. K. Law, Combustion Physics (Cambridge University Press, Cambridge, 2006). 
[22] L. Landau and E. Lifshitz, Fluid Mechanics (Pergamon, Oxford, 1989).

[23] J. D. Ott, E. S. Oran and J. D. Anderson, Jr., AIAA J. 41, 1391 (2003).

[24] Ya. B. Zeldovich and Yu. P. Raizer, Physics of Shock Waves and High-Temperature Hydrodynamic Phenomena (Dover, Mineola, NY, 2002).
[25] E. del Barco, A. D. Kent, S. Hill, J. M. North, N. S. Dalal, E. M. Rumberger, D. N. Hendrickson, N. Chakov, and G. Christou, J. Low Temp. Phys. 140, 119 (2005).

[26] P. Bruno, Phys. Rev. Lett. 96, 117208 (2006).

[27] D. Villuendas, D. Gheorghe, A. Hernández-Mínguez, F. Macià, J. M. Hernàndez, J. Tejada, R. J. Wijngaarden, Europhys. Lett. 84, 67010 (2008). 